\title{
A Random Attractor Family of the High Order Beam Equations with White Noise
}

\author{
Guoguang Lin, Jie Liu \\ School of Mathematics and Statistics, Yunnan University, Kunming, China \\ Email: 15925159599@163.com
}

How to cite this paper: Lin, G.G. and Liu, J. (2020) A Random Attractor Family of the High Order Beam Equations with White Noise. International Journal of Modern Nonlinear Theory and Application, 9, 51-61. https://doi.org/10.4236/ijmnta.2020.93004

Received: August 13, 2020

Accepted: September 15, 2020

Published: September 18, 2020

Copyright $\odot 2020$ by author(s) and Scientific Research Publishing Inc. This work is licensed under the Creative Commons Attribution International License (CC BY 4.0).

http://creativecommons.org/licenses/by/4.0/

\begin{abstract}
In this paper, we studied a class of damped high order Beam equation stochastic dynamical systems with white noise. First, the Ornstein-Uhlenbeck process is used to transform the equation into a noiseless random equation with random variables as parameters. Secondly, by estimating the solution of the equation, we can obtain the bounded random absorption set. Finally, the isomorphism mapping method and compact embedding theorem are used to obtain the system. It is progressively compact, then we can prove the existence of random attractors.
\end{abstract}

\section{Keywords}

Beam Type Equation, Random Attractor, White Noise

\section{Introduction}

We studied the random high order Beam equation with strong damping and white noise in this paper.

$$
\begin{gathered}
u_{t t}+\beta(-\Delta)^{2 m} u_{t}+M\left(\left\|D^{m} u\right\|_{p}^{p}\right) u_{t}+N\left(\left\|D^{m} u\right\|_{p}^{p}\right)(-\Delta)^{m} u+\alpha \Delta^{2 m} u=q(x) \dot{W}, \\
u(x, t)=0, \frac{\partial^{i} u}{\partial v^{i}}=0, i=1,2, \cdots, 2 m, \quad x \in D, \quad t \in[0,+\infty), \\
u(x, 0)=u_{0}(x), u_{t}(x, 0)=u_{1}(x) .
\end{gathered}
$$

where $M\left(\left\|D^{m} u\right\|_{p}^{p}\right) u_{t}, N\left(\left\|D^{m} u\right\|_{p}^{p}\right)(-\Delta)^{m} u$ are given functions, $m \geq 1, q \mathrm{~d} W$ describe a addable white noise, $\Omega$ denotes a area which bounded with smooth homogeneous Dirichlet boundary, $\partial \Omega$ denotes the boundary of $\Omega . \alpha, \beta$ are constants, $\alpha \Delta^{2 m} u, \beta(-\Delta)^{2 m} u_{t}$ are strong damping terms, $W(t)$ denotes a one-dimensional two-sided Wiener process on a probability space $(\Omega, F, P)$, $\Omega=\{\omega \in C(R, R): \omega(0)=0\}, F$ denotes a Borel $\sigma$-algebra generated by com- 
pact-open topology on $\Omega, P$ denotes a probability measure.

Random attractor is a random collection, which is measurable, compact, constant, and attract all the orbits. If it exists, it will be the smallest absorbing sets of system solution concentration, it's also the biggest invariant set. In a sense, random attractor is reminded as a reasonable extension of the global attractor to the classical dynamical system. Recently, more and more scholars have focused on the random dynamic system.

Guo [1] wrote a book about the random infinite dimensional dynamical system, it's the first book at home. It includes any experience of the author with random dynamic study and some research results, the latest development and results also be introduced.

Lin [2] studied the existence of stochastic attractors of high order nonlinear Beam equation.

$$
\mathrm{d} u_{t}+\left[(-\Delta)^{m} u_{t}+\phi\left(\left\|D^{m} u\right\|^{2}\right)(-\Delta)^{m} u_{t}+g(u)\right] \mathrm{d} t=q(x) \mathrm{d} W(t) .
$$

Qin [3] proved the random attractor for stochastic Beam equations with addable white noise, $\mathrm{Xu}$ [4] studied the non-autonomous stochastic wave equation with dispersion and dissipation terms.

$$
u_{t t}-\Delta u-\alpha \Delta u_{t}-\beta u_{t t}+h(u) u_{t}+\lambda u+f(x, u)=g(x, t) u+\varepsilon u \cdot \frac{\mathrm{d} W}{\mathrm{~d} t} .
$$

Crauel and Flandoli [5] studied the random attractor of the infinite dimensional equation. Cai and Fan [6] considered the dissipative KDV equation with multiplicative noise.

$$
\mathrm{d} u=\left(a u_{x x x x}+u_{x x}+\beta u u_{x x}+r u\right) \mathrm{d} t=f(x) \mathrm{d} t+b u \mathrm{~d} W(t), x \in D, t>0 .
$$

For more relevant studies, it can be referred to references in [7]-[12].

\section{Preliminaries}

In this section, some symbols and assumptions are introduced for convenience.

Let the operator $A=\Delta$ with Dirichlet boundary condition be selfadjoint, positive definite and linear. Set the eigenvalue of $A$ is $\left\{\lambda_{i}\right\}_{i \in N}$, and satisfies

$$
0<\lambda_{1} \leq \lambda_{2} \leq \cdots \leq \lambda_{m} \leq \cdots, \text { when } m \rightarrow+\infty, \lambda_{m} \rightarrow+\infty \text {. }
$$

Among them

$$
\frac{2 n}{n+2 m} \leq p \begin{cases}<\frac{2 n}{n-2 m}, & n>2 m \\ <\infty, & n \leq 2 m\end{cases}
$$

Set $E_{k}=H_{0}^{2 m+k}(\Omega) \times H_{0}^{k}(\Omega), k=1,2, \cdots, 2 m$ and define a weighted inner product and norm in $E_{k}$

$$
\begin{gathered}
\left(y_{1}, y_{2}\right)_{E_{k}}=\left(D^{2 m+k} u_{1}, D^{2 m+k} u_{2}\right)+\left(D^{k} v_{1}, D^{k} v_{2}\right), \\
\forall y_{i}=\left(u_{i}, v_{i}\right)^{\mathrm{T}}, \quad y=(u, v)^{\mathrm{T}} \in E_{k}, \quad i=1,2
\end{gathered}
$$

and 


$$
0<\varepsilon<\min \left\{\frac{\alpha-1}{\beta}, \frac{(\alpha-2) \beta \lambda_{1}^{2 m}}{\left(\beta^{2}-1\right) \lambda_{1}^{2 m}-4 M^{2}\left(\left\|D^{m} u\right\|_{p}^{p}\right)}\right\}, \beta>\frac{\alpha-2}{\varepsilon} .
$$

Definition 2.1 [2] Set $\left(\Omega, F, P,\left(\theta_{t}\right)_{t \in R}\right)$ is a metric dynamic system, if $B\left(R^{+}\right) \times F \times B(X)$, measurable mapping

$$
S: R^{+} \times \Omega \times X \rightarrow X, \quad(t, w, x) \mapsto S(t, w, x),
$$

satisfy

1) For all $s, t \geq 0$ and $\omega \in \Omega$, mapping $S(t, w):=S(t, w, \cdot)$ satisfy

$$
S(0, w)=i d, \quad S(t+s, w)=S\left(t, \theta_{s} w\right) \circ S(s, w) ;
$$

2) For every $w \in \Omega$, mapping $(t, w) \mapsto S(t, w, x)$ continuous.

It is said that $S$ is a continuous random dynamic system on $\left(\Omega, F, P,\left(\theta_{t}\right)_{t \in R}\right)$.

Definition 2.2 [2] It is said that the random set $B(w) \subset X$ is slowly increasing, if $w \in \Omega, \beta \geq 0$, there is

$$
\lim _{|s| \rightarrow \infty} \inf \mathrm{e}^{-\beta s} d\left(B\left(\theta_{-s} w\right)\right)=0 .
$$

and $d(B)=\sup _{x \in B}\|x\|_{X}$, for all $x \in X$.

Definition 2.3 [2] $D(w)$ denotes a collection of all random sets on $X$, random set $B_{0}(w)$ denotes a absorption set on $D(w)$, if for every $B(w) \in D(w)$ and $P-a$ e. $w \in \Omega$, there is $T_{B}(w)>0$ make

$$
S\left(t, \theta_{-t} \omega\right)\left(B\left(\theta_{-t} \omega\right)\right) \subset B_{0}(\omega) .
$$

Definition 2.4 [2] The random set $A(w)$ becomes the random attractor of the continuous random dynamic system $S(t)$ on $X$. If the random set $A(w)$ satisfies:

1) $A(w)$ is a random compact set;

2) $A(w)$ is a invariant set, for every $t>0, S(t, w) A(w)=A\left(\theta_{t} w\right)$;

3) $A(\omega)$ attracts all sets on $D(w)$, for any $B(w) \in D(w)$ and $P-a e \omega \in \Omega$, we have the limit formula:

$$
\lim _{t \rightarrow \infty} d\left(S\left(t, \theta_{-t} w\right)\left(B\left(\theta_{-t} w\right)\right), A(w)\right)=0
$$

$$
d(A, B)=\sup _{x \in A} \inf _{y \in B}\|x-y\|_{H} \text { denotes the Hausdorff half distance. }(A, B \subseteq H) .
$$

Definition 2.5 [2] Random set $B_{k}(w) \in D(w)$ is the random absorption set of the random dynamic system $(s(t, w))_{t \geq 0}$, and the random set $B_{k}(w)$ satisfies

1) Random set $B_{k}(w)$ is a closed set on Hilbert space $X$;

2) For $P$-ae. $w \in \Omega$, random set $B_{k}(w)$ meet the following progressive compactness conditions: For any sequence $x_{n} \in s\left(t_{n}, \theta_{-t_{n}} w\right) B_{k}\left(\theta_{-t_{n}} w\right)$ in $t_{n} \rightarrow+\infty$, there is a convergent subsequence in space $X$. Then the stochastic dynamic system $(s(t, w))_{t \geq 0}$ has a unique global attractor

$$
A_{k}(w)=\bigcap_{\tau \geq t_{k}(w) t \geq \tau} \overline{\bigcup_{t} s\left(t, \theta_{-t} w\right) B_{k}\left(\theta_{-t} w\right)}
$$




\section{Existence of Random Attractors}

\subsection{Existence and Uniqueness of Solution}

For convenience, Equations (1)-(3) can be reduced to

$$
\left\{\begin{array}{l}
\mathrm{d} u=u_{t} \mathrm{~d} t \\
\mathrm{~d} u_{t}+\left(\beta(-\Delta)^{2 m} u_{t}+M\left(\left\|D^{m} u\right\|_{p}^{p}\right) u_{t}+N\left(\left\|D^{m} u\right\|_{p}^{p}\right)(-\Delta)^{m} u+\alpha \Delta^{2 m} u\right) \mathrm{d} t \\
=q(x) \mathrm{d} W(t) \\
t \in[0,+\infty) \\
u(x, 0)=u_{0}(x), u_{t}(x, 0)=u_{1}(x), \quad x \in D .
\end{array}\right.
$$

Set $\phi=(u, y)^{\mathrm{T}}, \quad y=u_{t}+\varepsilon u$, then Equation (1) is equivalent to the following stochastic differential equation

$$
\left\{\begin{array}{l}
\mathrm{d} \phi+L \phi \mathrm{d} t=F\left(\theta_{t} \omega, \phi\right), \\
\phi_{0}(\omega)=\left(u_{0}, u_{1}+\varepsilon u_{0}\right)^{\mathrm{T}} .
\end{array}\right.
$$

and

$$
\begin{aligned}
& \phi=\left(\begin{array}{l}
u \\
y
\end{array}\right), \quad F\left(\theta_{t} \omega, \phi\right)=\left(\begin{array}{c}
0 \\
q(x) \mathrm{d} W(t)
\end{array}\right) \\
& L=\left(\begin{array}{cc}
\varepsilon I & -I \\
\left((\alpha-\beta \varepsilon) A^{2 m}+N\left(\left\|D^{m} u\right\|_{p}^{p}\right) A^{m}+\varepsilon^{2}-\varepsilon M\left(\left\|D^{m} u\right\|_{p}^{p}\right)\right) I & \left(\beta A^{2 m}+M\left(\left\|D^{m} u\right\|_{p}^{p}\right)-\varepsilon\right) I
\end{array}\right) \\
& \delta=\delta\left(\theta_{t} \omega\right)=-\int_{-\infty}^{0} \mathrm{e}^{s} \theta_{t} \omega(s) \mathrm{d} s,
\end{aligned}
$$

$\delta\left(\theta_{t} \omega\right)$ denotes a Ornstein-Uhlenbeck process, it is a stationary solution of Itô equation

$$
\mathrm{d} \delta+\delta \mathrm{d} t=\mathrm{d} W
$$

$v=y-q(x) \delta\left(\theta_{t} \omega\right)$, then Equation (14) can be reduced to

$$
\left\{\begin{array}{l}
\mathrm{d} \varphi+L \phi \mathrm{d} t=\bar{F}\left(\theta_{t} \omega, \varphi\right), \\
\varphi_{0}(\omega)=\left(u_{0}, u_{1}+\varepsilon u_{0}-q(x) \delta\left(\theta_{t} \omega\right)\right)^{\mathrm{T}} .
\end{array}\right.
$$

And

$$
\begin{aligned}
& \varphi=\left(\begin{array}{l}
u \\
v
\end{array}\right) \\
& \bar{F}\left(\theta_{t} \omega, \varphi\right)=\left(\begin{array}{c}
q(x) \delta\left(\theta_{t} \omega\right) \\
q(x) \mathrm{d} W(t)+\left(\varepsilon-\beta A^{2 m}-1-M\left(\left\|D^{m} u\right\|_{p}^{p}\right)\right) q(x) \delta\left(\theta_{t} \omega\right)
\end{array}\right), \\
& L=\left(\begin{array}{cc}
\varepsilon I & -I \\
\left((\alpha-\beta \varepsilon) A^{2 m}+N\left(\left\|D^{m} u\right\|_{p}^{p}\right) A^{m}+\varepsilon^{2}-\varepsilon M\left(\left\|D^{m} u\right\|_{p}^{p}\right)\right) I & \left(\beta A^{2 m}+M\left(\left\|D^{m} u\right\|_{p}^{p}\right)-\varepsilon\right) I
\end{array}\right) .
\end{aligned}
$$




\subsection{The Existence of Random Attractors}

This section mainly considers existence of the random attractor of problem (1). First, we can prove that the random dynamic system $S(t, \omega)$ has a bounded random absorption set. For this reason, all slowly increasing subsets in the space $E$ are denoted as $D(E)$.

Lemma $1 E_{k}=H_{0}^{2 m+k}(\Omega) \times H_{0}^{k}(\Omega)$, for every $y=\left(y_{1}, y_{2}\right)^{\mathrm{T}} \in E_{k}$, $(k=1,2, \cdots, 2 m)$, When

$$
\begin{aligned}
& k_{1}=\min \left\{\varepsilon+\frac{\beta \varepsilon+1-\alpha}{2 \beta}+\frac{\varepsilon\left(\varepsilon^{2}-\varepsilon M\left(\left\|D^{m} u\right\|_{p}^{p}\right)\right)}{2 \lambda_{1}^{2 m}},\right. \\
& \left.\frac{\varepsilon-M\left(\left\|D^{m} u\right\|_{p}^{p}\right)}{2}+\frac{\beta \lambda_{1}^{2 m}}{2}-\varepsilon+M\left(\left\|D^{m} u\right\|_{p}^{p}\right)\right\}, k_{2}=\frac{\beta(\beta \varepsilon+2-\alpha)}{2} .
\end{aligned}
$$

Then

$$
(L y, y)_{E_{k}} \geq k_{1}\|y\|_{E_{k}}^{2}+k_{2}\left\|D^{2 m+k} y_{2}\right\|^{2}
$$

\section{Proof}

$$
\begin{aligned}
& (L y, y)_{E_{k}} \\
= & \left(D^{2 m+k}\left(\varepsilon y_{1}-y_{2}\right), D^{2 m+k} y_{1}\right)+\left(D ^ { k } \left((\alpha-\beta \varepsilon) A^{2 m} y_{1}+N\left(\left\|D^{m} u\right\|_{p}^{p}\right) A^{m} y_{1}\right.\right. \\
& \left.\left.+\beta A^{2 m} y_{2}\left(\varepsilon^{2}-\varepsilon M\left(\left\|D^{m} u\right\|_{p}^{p}\right)\right) y_{1}+M\left(\left\|D^{m} u\right\|_{p}^{p}\right) y_{2}\right), D^{k} y_{2}\right) \\
= & \varepsilon\left\|D^{2 m+k} y_{1}\right\|^{2}+(\alpha-\beta \varepsilon-1)\left(D^{2 m+k} y_{1}, D^{2 m+k} y_{2}\right) \\
& +N\left(\left\|D^{m} u\right\|_{p}^{p}\right)\left(D^{2 m+k} y_{1}, D^{k} y_{2}\right)+\beta\left\|D^{2 m+k} y_{2}\right\|^{2} \\
& +\left(\varepsilon^{2}-\varepsilon M\left(\left\|D^{m} u\right\|_{p}^{p}\right)\right)\left(D^{k} y_{1}, D^{k} y_{2}\right)+\left(M\left(\left\|D^{m} u\right\|_{p}^{p}\right)-\varepsilon\right)\left\|D^{k} y_{2}\right\|^{2} \\
\geq & \varepsilon\left\|D^{2 m+k} y_{1}\right\|^{2}+\frac{\beta \varepsilon+1-\alpha}{2 \beta}\left\|D^{2 m+k} y_{1}\right\|^{2}+\frac{\beta(\beta \varepsilon+1-\alpha)}{2}\left\|D^{2 m+k} y_{2}\right\|^{2} \\
& +\frac{\varepsilon^{2}-\varepsilon M\left(\left\|D^{m} u\right\|_{p}^{p}\right)}{2}\left\|D^{k} y_{1}\right\|^{2}+\frac{\varepsilon^{2}-\varepsilon M\left(\left\|D^{m} u\right\|_{p}^{p}\right)}{2}\left\|D^{k} y_{2}\right\|^{2} \\
& +\frac{\beta}{2}\left\|D^{2 m+k} y_{2}\right\|^{2}+\frac{\beta \lambda_{1}^{2 m}}{2}\left\|D^{k} y_{2}\right\|^{2}+\left(M\left(\left\|D^{m} u\right\|_{p}^{p}\right)-\varepsilon\right)\left\|D^{k} y_{2}\right\|^{2} \\
= & \left(\varepsilon+\frac{\beta \varepsilon+1-\alpha}{2 \beta}+\frac{\varepsilon\left(\varepsilon^{2}-\varepsilon M\left(\left\|D^{m} u\right\|_{p}^{p}\right)\right)}{2 \lambda_{1}^{2 m}}\left\|D^{2 m+k} y_{1}\right\|^{2}\right. \\
& +\left(\frac{\beta}{2}+\frac{\beta(\beta \varepsilon+1-\alpha)}{2}\left\|D^{2 m+k} y_{2}\right\|^{2}\right. \\
& +\left(\frac{\varepsilon-M\left(\left\|D^{m} u\right\|_{p}^{p}\right)}{2}+\frac{\beta \lambda_{1}^{2 m}}{2}+M\left(\left\|D^{m} u\right\|_{p}^{p}\right)-\varepsilon\right)\left\|D^{k} y_{2}\right\|^{2} \\
& \left(\frac{\beta}{2}\right)
\end{aligned}
$$




$$
\geq k_{1}\|y\|_{E_{k}}^{2}+k_{2}\left\|D^{2 m+k} y_{2}\right\|^{2}
$$

According to the Formula (7), we can get

$$
\begin{aligned}
& \frac{\beta}{2}+\frac{\beta(\beta \varepsilon+1-\alpha)}{2} \geq 0, \\
& \frac{\varepsilon-M\left(\left\|D^{m} u\right\|_{p}^{p}\right)}{2}+\frac{\beta \lambda_{1}^{2 m}}{2}+M\left(\left\|D^{m} u\right\|_{p}^{p}\right)-\varepsilon \\
& =\frac{\beta \lambda_{1}^{2 m}}{2}-\varepsilon+\frac{\varepsilon+M\left(\left\|D^{m} u\right\|_{p}^{p}\right)}{2} \geq 0 .
\end{aligned}
$$

So set

$$
\begin{aligned}
& k=\min \left\{\varepsilon+\frac{\beta \varepsilon+1-\alpha}{2 \beta}+\frac{\varepsilon\left(\varepsilon^{2}-\varepsilon M\left(\left\|D^{m} u\right\|_{p}^{p}\right)\right)}{2 \lambda_{1}^{2 m}},\right. \\
& \left.\frac{\varepsilon-M\left(\left\|D^{m} u\right\|_{p}^{p}\right)}{2}+\frac{\beta \lambda_{1}^{2 m}}{2}-\varepsilon+M\left(\left\|D^{m} u\right\|_{p}^{p}\right) \varepsilon\right\}, \\
& k_{2}=\frac{\beta(\beta \varepsilon+2-\alpha)}{2} .
\end{aligned}
$$

Lemma $2 \phi$ denotes a solution of problem (14), then there is a bounded random compact set $\tilde{B}_{0 k}(\omega) \in D\left(E_{k}\right)$, so that there is a random variable $T_{B_{k}(\omega)}>0$ for any slowly increasing random set $B(\omega) \in D\left(E_{k}\right)$, such that

$$
\phi\left(t, \theta_{-t} \omega\right) B\left(\theta_{-t} \omega\right) \subset \tilde{B}_{0 k}(\omega), \forall t \geq T_{B_{k}}(\omega), \omega \in \Omega .
$$

Proof $\varphi$ denotes a solution of problem (16), use $\varphi=(u, v)^{\mathrm{T}} \in E_{k}$ to take the inner product with the Equation (16), we obtain

$$
\frac{1}{2} \frac{\mathrm{d}}{\mathrm{d} t}\|\varphi\|_{E_{k}}^{2}+(L \varphi, \varphi)_{E_{k}}=\left(\bar{F}\left(\theta_{t} \omega, \varphi\right), \varphi\right) .
$$

From Lemma 1

$$
(L \varphi, \varphi)_{E_{k}} \geq k_{1}\|\varphi\|_{E_{k}}^{2}+k_{2}\left\|D^{2 m+k} v\right\|^{2}
$$

and

$$
\begin{aligned}
& \left(\bar{F}\left(\theta_{t} \omega, \varphi\right), \varphi\right) \\
= & \left(D^{2 m+k} q(x) \delta\left(\theta_{t} \omega\right), D^{2 m+k} u\right) \\
& +\left(\varepsilon-\beta A^{2 m}-1-M\left(\left\|D^{m} u\right\|_{p}^{p}\right)\right)\left(D^{k} q(x) \delta\left(\theta_{t} \omega\right), D^{k} v\right) \\
\leq & \left(\frac{\varepsilon}{2}+\frac{\left(1+M\left(\left\|D^{m} u\right\|_{p}^{p}\right)\right)^{2}}{2 \varepsilon}\right)\left\|D^{k} q(x)\right\|^{2}\left|\delta\left(\theta_{t} \omega\right)\right|^{2} \\
& +\left(\frac{\varepsilon}{2}+\frac{2 \varepsilon M^{2}\left(\left\|D^{m} u\right\|_{p}^{p}\right.}{\lambda_{1}^{2 m}}\right)\left\|D^{2 m+k} v\right\|^{2}+\left(\frac{\beta^{2}}{2 \varepsilon}+\frac{1}{2}\right)
\end{aligned}
$$




$$
\left\|D^{2 m+k} q(x)\right\|^{2}\left|\delta\left(\theta_{t} \omega\right)\right|^{2}+\frac{1}{2}\left\|D^{2 m+k} u\right\|^{2} .
$$

From Equations (22) and (23), Equation (21) can be written as

$$
\begin{aligned}
& \frac{\mathrm{d}}{\mathrm{d} t}\|\varphi\|_{E_{k}}^{2}+2 k_{1}\|\varphi\|_{E_{k}}^{2}+\left(2 k_{2}-\varepsilon-\frac{4 \varepsilon M^{2}\left(\left\|D^{m} u\right\|_{p}^{p}\right)}{\lambda_{1}^{2 m}}\right)\left\|D^{2 m+k} v\right\|^{2} \\
& \leq\left(\varepsilon+\frac{\left(1+M\left(\left\|D^{m} u\right\|_{p}^{p}\right)\right)^{2}}{\varepsilon}\right)\left\|D^{k} q(x)\right\|^{2}\left|\delta\left(\theta_{t} \omega\right)\right|^{2} \\
& +\left(\frac{\beta^{2}}{\varepsilon}+1\right)\left\|D^{2 m+k} q(x)\right\|^{2}\left|\delta\left(\theta_{t} \omega\right)\right|^{2}+\left\|D^{2 m+k} u\right\|^{2}
\end{aligned}
$$

Set

$$
\begin{aligned}
C_{1}= & \left\|D^{2 m+k} u\right\|^{2}, \eta=2 k_{1}, \\
P_{1}= & \left(\varepsilon+\frac{\left(1+M\left(\left\|D^{m} u\right\|_{p}^{p}\right)\right)^{2}}{\varepsilon}\right)\left\|D^{k} q(x)\right\|^{2}\left|\delta\left(\theta_{t} \omega\right)\right|^{2} \\
& +\left(\frac{\beta^{2}}{\varepsilon}+1\right)\left\|D^{2 m+k} q(x)\right\|^{2}\left|\delta\left(\theta_{t} \omega\right)\right|^{2},
\end{aligned}
$$

then

$$
\frac{\mathrm{d}}{\mathrm{d} t}\|\varphi\|_{E_{k}}^{2}+\eta\|\varphi\|_{E_{k}}^{2} \leq P_{1}+C_{1}
$$

According to the Formula (7), we can get

$$
2 k_{2}-\varepsilon-\frac{4 \varepsilon M^{2}\left(\left\|D^{m} u\right\|_{p}^{p}\right)}{\lambda_{1}^{2 m}}=\beta(\beta \varepsilon+2-\alpha)-\varepsilon-\frac{4 \varepsilon M^{2}\left(\left\|D^{m} u\right\|_{p}^{p}\right)}{\lambda_{1}^{2 m}}>0 .
$$

From the Gronwall's inequality, $P$-a.e. $\omega \in \Omega$, we have

$$
\|\varphi(t, \omega)\|_{E_{k}}^{2} \leq \mathrm{e}^{-\eta t}\left\|\varphi_{0}(\omega)\right\|_{E_{k}}^{2}+\int_{0}^{t} \mathrm{e}^{-\eta(t-r)}\left(C_{1}+P_{1}\left\|\delta\left(\theta_{t} \omega\right)\right\|^{2}\right) \mathrm{d} r,
$$

because $\delta\left(\theta_{t} \omega\right)$ is slowly increasing, and $\delta\left(\theta_{t} \omega\right)$ is continuous with respect to $t$, according to the literature [3], a slowly increasing random variable $r_{1}: \Omega \rightarrow R^{+}$can be obtained, so for $\forall t \in R, \omega \in \Omega$ there is

$$
\left|\delta\left(\theta_{t} \omega\right)\right|^{2} \leq r_{1}\left(\theta_{t} \omega\right) \leq \mathrm{e}^{\frac{n}{2} t} r_{1}(\omega) .
$$

Substituting $\theta_{-t} \omega$ for $\omega$ in (27), we get

$$
\left\|\varphi\left(t, \theta_{-t} \omega\right)\right\|_{E_{k}}^{2} \leq \mathrm{e}^{-\eta t}\left\|\varphi_{0}\left(\theta_{-t} \omega\right)\right\|_{E_{k}}^{2}+\int_{0}^{t} \mathrm{e}^{-\eta(t-r)}\left(C_{1}+P_{1}\left\|\delta\left(\theta_{-t} \omega\right)\right\|^{2}\right) \mathrm{d} r,
$$

and

$$
\int_{0}^{t} \mathrm{e}^{-\eta(t-r)}\left(C_{1}+p_{1}\left|\delta\left(\theta_{r-t} \omega\right)\right|^{2}\right) \mathrm{d} r=\int_{0}^{t} \mathrm{e}^{\eta \tau}\left(C_{1}+p_{1}\left|\delta\left(\theta_{\tau} \omega\right)\right|^{2}\right) \mathrm{d} r \leq \frac{C_{1}}{\eta}+\frac{2}{\eta} p_{1} r_{1}(\omega) .
$$


Because $\phi_{0}\left(\theta_{-t} \omega\right) \in B_{k}\left(\theta_{-t} \omega\right)$ is slowly increasing, and $\left|\delta\left(\theta_{-t} \omega\right)\right|$ is also slowly increasing, so let

$$
R_{0}^{2}(\omega)=\frac{C_{1}}{\eta}+\frac{2}{\eta} p_{1} r_{1}(\omega) .
$$

Then $R_{0}^{2}(\omega)$ also slowly increasing, $\hat{B}_{0 k}=\left\{\varphi \in E_{k}:\|\varphi\|_{E_{k}} \leq R_{0}(\omega)\right\}$ denotes a random absorption set, because

$$
\begin{aligned}
& \tilde{S}\left(t, \theta_{-t} \omega\right) \varphi_{0}\left(\theta_{-t} \omega\right) \\
& =\phi\left(t, \theta_{-t} \omega\right)\left(\varphi_{0}\left(\theta_{-t} \omega\right)+\left(0, q(x) \delta\left(\theta_{-t} \omega\right)\right)^{\mathrm{T}}\right)-(0, q(x) \delta(\omega))^{\mathrm{T}},
\end{aligned}
$$

so

$$
\tilde{B}_{0 k}(\omega)=\left\{\phi \in E_{k}:\|\phi\|_{E_{k}} \leq R_{0}(\omega)+\left\|D^{k} q(x) \delta(\omega)\right\|=\bar{R}_{0}(\omega)\right\} .
$$

Then $\tilde{B}_{0 k}(\omega)$ is the random absorption set of $\phi(t, \omega)$, and $\tilde{B}_{0 k}(\omega) \in D\left(E_{k}\right)$. So the lemma is proved.

Lemma 3 When $k=m$, for any $B_{m}(\omega) \in D\left(E_{m}\right), \phi(t)$ is the solution of Equation (14) under the initial value condition $\phi_{0}=\left(u_{0}, u_{1}+\varepsilon u_{0}\right)^{\mathrm{T}} \in B_{m}$. It can be decomposed into $\phi=\phi_{1}+\phi_{2}$, where $\phi_{1}$ and $\phi_{2}$ satisfy

$$
\begin{aligned}
& \left\{\begin{array}{l}
\mathrm{d} \phi_{1}+L \phi_{1} \mathrm{~d} t=0 \\
\phi_{10}(\omega)=\left(u_{0}, u_{1}+\varepsilon u_{0}\right)^{\mathrm{T}}
\end{array}\right. \\
& \left\{\begin{array}{l}
\mathrm{d} \phi_{2}+L \phi_{2} \mathrm{~d} t=F(\omega, \phi) \\
\phi_{20}(\omega)=0
\end{array}\right.
\end{aligned}
$$

Then

$$
\left\|\phi_{1}\left(t, \theta_{-t} \omega\right)\right\|_{E_{m}}^{2} \rightarrow 0(t \rightarrow \infty), \quad \forall \phi_{0}\left(\theta_{-t} \omega\right) \in B\left(\theta_{-t} \omega\right),
$$

and there is a slowly increasing random radius $R_{1}(\omega)$, so that for every $\omega \in \Omega$, satisfy

$$
\left\|\phi_{2}\left(t, \theta_{-t} \omega\right)\right\|_{E_{m}}^{2} \leq R_{1}(\omega)
$$

Proof $\varphi=\varphi_{1}+\varphi_{2}=\left(u_{1}, u_{1 t}+\varepsilon u_{1}\right)^{\mathrm{T}}+\left(u_{2}, u_{2 t}+\varepsilon u_{2}-q(x) \delta\left(\theta_{t} \omega\right)\right)^{\mathrm{T}}$ is a solution of Equation (16), from Equations (33) and (34), it can be seen that $\varphi_{1}$ and $\varphi_{2}$ satisfy

$$
\begin{aligned}
& \left\{\begin{array}{l}
\varphi_{1 t}+L \varphi_{1} \mathrm{~d} t=0, \\
\varphi_{10}=\varphi_{0}=\left(u_{0}, u_{1}+\varepsilon u_{0}\right)^{\mathrm{T}},
\end{array}\right. \\
& \left\{\begin{array}{l}
\varphi_{2 t}+L \varphi_{2} \mathrm{~d} t=F(\omega, \varphi) \\
\varphi_{20}=0
\end{array}\right.
\end{aligned}
$$

Using $\varphi_{1}=\left(u_{1}, u_{1 t}+\varepsilon u_{1}\right)^{\mathrm{T}}$ and Equation (37) to take the inner product, we get

$$
\frac{1}{2} \frac{\mathrm{d}}{\mathrm{d} t}\left\|\varphi_{1}\right\|_{E_{m}}^{2}+\left(L \varphi_{1}, \varphi_{1}\right)_{E_{m}}=0
$$


according to lemma 2 and the Gronwall's inequality, we obtain

$$
\left\|\varphi_{1}(t, \omega)\right\|_{E_{m}}^{2} \leq \mathrm{e}^{-2 k_{1} t}\left\|\varphi_{0}(\omega)\right\|_{E_{m}}^{2},
$$

replace $\omega$ in (40) with $\theta_{-t} \omega$, and because $\delta\left(\theta_{-t} \omega\right) \in B$ is slowly increasing, then

$$
\left\|\varphi_{1}\left(t, \theta_{-t} \omega\right)\right\|_{E_{m}}^{2} \leq \mathrm{e}^{-2 k_{1} t}\left\|\varphi_{0}\left(\theta_{-t} \omega\right)\right\|_{E_{m}}^{2} \rightarrow 0(t \rightarrow \infty), \quad \forall \varphi_{0}\left(\theta_{-t} \omega\right) \in B_{m}
$$

Using $\varphi_{2}=\left(u_{2}, u_{2 t}+\varepsilon u_{2}-q(x) \delta\left(\theta_{t} \omega\right)\right)^{\mathrm{T}}$ and Equation (38) to take the inner product, we get

$$
\frac{\mathrm{d}}{\mathrm{d} t}\left\|\varphi_{2}\right\|_{E_{m}}^{2}+\eta\left\|\varphi_{2}\right\|_{E_{m}}^{2} \leq C_{2}+P_{2}\left|\delta\left(\theta_{t} \omega\right)\right|^{2}
$$

and $P_{2}=P_{1}\left|\delta\left(\theta_{t} \omega\right)\right|^{2}$.

According to lemma 1, lemma 2, Equation (29), the Gronwall's inequality, and replace $\omega$ with $\theta_{-t} \omega$, we obtain

$$
\begin{aligned}
\left\|\varphi^{2}\left(t, \theta_{-t} \omega\right)\right\|_{E_{m}}^{2} & \leq \mathrm{e}^{-\eta t}\left\|\phi_{20}\left(\theta_{-t} \omega\right)\right\|_{E_{m}}^{2}+\int_{0}^{t} \mathrm{e}^{-\eta(t-r)}\left(C_{2}+P_{2}\left|\delta\left(\theta_{r-t} \omega\right)\right|^{2}\right) \mathrm{d} r \\
& \leq \frac{C_{3}}{\eta}+\frac{2}{\eta} P_{2} r_{1}(\omega),
\end{aligned}
$$

set

$$
R_{1}^{2}(\omega)=\frac{C_{3}}{\eta}+\frac{2}{\eta} p_{2} r_{1}(\omega)
$$

for every $\omega \in \Omega$,

$$
\left\|\phi^{2}\left(t, \theta_{-t} \omega\right)\right\|_{E_{m}} \leq R_{1}(\omega)
$$

and $R_{1}(\omega)$ is slowly increasing. The lemma is proved.

Lemma 4 The stochastic dynamic system $\{S(t, \omega), t \geq 0\}$ determined by Equation (17) has a compact absorption set $K(\omega) \subset E_{k}$ under condition $t=0, \quad P-$ a.e. $\omega \in \Omega$

Proof Suppose $K(\omega)$ is a closed sphere with $R_{1}(\omega)$ as the radius in space $D\left(A^{\frac{3}{4}}\right) \times D\left(A^{\frac{1}{4}}\right)$. According to the embedding relationship $E_{k} \subset E_{0}, K(\omega)$ is a compact set in $E_{k}$. For any slowly increasing random set $B_{k}(\omega)$ in $E$, for $\forall \phi\left(t, \theta_{-t} \omega\right) \in B_{k}$, according to Lemma 3.1, there is $\varphi_{2}=\varphi-\varphi_{1} \in K(\omega)$, so for every $t \geq T_{B_{k}(\omega)}>0$,

$$
\begin{aligned}
& d_{E_{k}}\left(S\left(t, \theta_{-t} \omega\right) B_{k}\left(\theta_{-t} \omega\right), K(\omega)\right) \\
& =\inf _{\vartheta(t) \in K(\omega)}\left\|\varphi\left(t, \theta_{-t} \omega\right)-\vartheta(t)\right\|_{E_{k}}^{2} \leq\left\|\varphi\left(t, \theta_{-t} \omega\right)\right\|_{E_{k}}^{2} \\
& \leq \mathrm{e}^{-\eta t}\left\|\varphi_{0}\left(\theta_{-t} \omega\right)\right\|_{E_{k}}^{2} \rightarrow 0, \quad(t \rightarrow \infty)
\end{aligned}
$$

Therefore, for any slowly increasing random set $B_{k}(\omega)$ in $E_{k}$, there is

$$
d_{E_{k}}\left(S\left(t, \theta_{-t} \omega\right) B\left(\theta_{-t} \omega\right), K(\omega)\right) \rightarrow 0, \quad t \rightarrow \infty, \omega \in \Omega .
$$


According to Lemma 1 to Lemma 4, there is the following theorem.

Theorem 1 Random dynamic system $\{S(t, \omega), t \geq 0\}$ has a random attractor $A_{k}(\omega) \subset K(\omega) \subset E_{k}, \omega \in \Omega$, and there is a slowly increasing random set $K(\omega)$, Pa.e. $\omega \in \Omega$,

$$
A_{k}(w)=\bigcap_{t \geq 0} \overline{\bigcup_{\tau \geq t} S\left(t, \theta_{-\tau} w\right) K\left(\theta_{-\tau} w\right)}
$$

and

$$
S(t, w) A_{k}(\omega)=A_{k}\left(\theta_{t} \omega\right)
$$

\section{Conclusion}

We studied a class of damped high order Beam equation stochastic dynamical systems with white noise, by using the Ornstein-Uhlenbeck process, estimating the solution of the equation and the isomorphism mapping method, then we can get the existence of the random attractor family, I wish there will be some more convenient methods can be shown off. Further we can make the inertial manifolds of the model.

\section{Conflicts of Interest}

The authors declare no conflicts of interest regarding the publication of this paper.

\section{References}

[1] Guo, B.L. (2000) Infinite Dimensional Dynamic System. National Defense Industry Press, Beijing.

[2] Lin, G., Chen, L. and Wang, W. (2017) Random Attractors of the Stochastuc Strongly Damped for the High Order Nonlinear Beam Type Equation. International Journal of Mordern Nonlinear Theory and Application, 6, 59-69. https://doi.org/10.4236/ijmnta.2017.62005

[3] Qin, C.L. and Du, J.J. (2016) Random Attractor for Strongly Damped Stochastic Beam Equation. Journal of Science of Teachers College and University, 36, 6-11.

[4] Xu, G.G., Wang, L.B. and Lin, G.G. (2017) Random Attractors for Nonautonomous Stochastic Wave Equations with Dispersive and Dissipative Terms. Journal of Applied Functional Analysis, 19, 131-149.

[5] Crauel, H. and Flandoli, F. (1994) Attractors for Random Dynamical Systems. Probability Theory and Related Fields, 100, 365-393. https://doi.org/10.1007/BF01193705

[6] Cai, D.M., Fan, X.M. and Ye, J.J. (2014) The Random Attractor of Dissipative KDV Type Equation with Multiplicative Noise Is Considered. Journal of Southwest University for Nationalities (Natural Science Edition), 40, 900-904.

[7] Hao, H.J.J. and Zhou, S.F. (2010) Existence of Random Attractors for Strongly Demoed Stochastic Sine-Gordon Equations. Journal of Shanghai Normal University (Natural Science Edition), 39, 121-127.

[8] Wang, R. and Li, Y.R. (2012) Random Attractor of Generalized Ginzburg-Landau Equation with Multiplicative White Noise. Journal of Southwest University for Nationalities (Natural Science Edition), 2, 34. 
[9] Cheng, Y.Y. and Li, Y.R. (2012) Random Attractor of Generalized Kuramoto-Sivashinsky Equation with Multiplicative White Noise. Journal of Southwest University for Nationalities (Natural Science Edition), 37, 27-30.

[10] Li, X.T. and Xu, L. (2014) Existence of Random Attractors for Stochastic Delay Discrete Wave Equations. Journal of Jilin University (Science Edition), 52, 261-262.

[11] Ban, A.L. (2018) Asymptotic Behavior of a Class of Stochastic Wave Equations. Journal of Anhui Normal University (Natural Science Edition), 41, 329-334.

[12] Zhao, C.D. and Zhou, S.F. (2009) Sufficient Conditions for the Existence of Global Random Attractors for Stochastic Lattice Dynamical Systems and Applications. Journal of Mathematical Analysis and Applications, 354, 78-95.

https://doi.org/10.1016/j.jmaa.2008.12.036 